\title{
Modeling and Evaluation of the Impact of Motorcycles Mobility on Vehicular Traffic
}

\author{
Adel Izadi, Ashkan Gholamhosseinian, Jochen Seitz \\ Communication Networks Group, Technische Universität Ilmenau, Ilmenau, Germany \\ Email: adel.izadi@tu-ilmenau.de, ashkan.gholamhosseinian@tu-ilmenau.de, jochen.seitz@tu-ilmenau.de
}

How to cite this paper: Izadi, A., Gholamhosseinian, A. and Seitz, J. (2021) Modeling and Evaluation of the Impact of Motorcycles Mobility on Vehicular Traffic. Journal of Transportation Technologies, 11, 426435 .

https://doi.org/10.4236/jtts.2021.113028

Received: May 20, 2021

Accepted: July 19, 2021

Published: July 22, 2021

Copyright $\odot 2021$ by author(s) and Scientific Research Publishing Inc. This work is licensed under the Creative Commons Attribution International License (CC BY 4.0).

http://creativecommons.org/licenses/by/4.0/

\begin{abstract}
Traffic simulation can help to evaluate the impact of different mobility behaviors on the traffic flow from safety, efficiency, and environmental views. The objective of this paper is to extend the SUMO (Simulation of Urban Mobility) road traffic simulator to model and evaluate the impact of motorcycles mobility on vehicular traffic. First, we go through diverse mobility aspects and models for motorcycles in SUMO. Later, we opt for the most suitable mobility models of motorcycles. Finally, the impact of motorcycle mobility on different kinds of vehicles is investigated in terms of environment, fuel consumption, velocity and travel time. The result of modeling and evaluation shows that based on the mobility model of the motorcycle, vehicular traffic flow can be enhanced or deteriorated.
\end{abstract}

\section{Keywords}

Traffic Simulation, SUMO, Motorcycle Mobility

\section{Introduction}

There have been many efforts to enhance the mobility modeling of vehicles in order to assess or replicate specific traffic conditions such as jams or crashes. With the advent of intelligent transport systems, numerous novel applications have been proposed concerning vehicular modeling in terms of both individual and overall traffic flow. In this context, due to the growing number of motorcycles in many countries, we must take into account the significance of motorcycles' and other two wheelers' role in vehicular mobility.

The improvement of traffic efficiency is a worldwide problem that many governments are dealing with especially on major roads and in big cities. Motorcycles are one of the most popular vehicles that have certain effects on the traffic flows and their popularity mainly comes from their ability to move around the 
traffic. Based on their size and abilities in mobility, motorcycles sometimes do not follow the same physical traffic rules as other vehicles. For example, they can accelerate or decelerate faster, maneuver between lanes or in a shared lane, move to adjacent lanes, or even form dense traffic on an unsaturated road. Thereby, they can affect traffic jams, efficiency, safety and congestion [1].

Nowadays, since the number of vehicle types is increasing, conducting research and studying on traffic flow issues is one of the interesting topics for many companies. Based on the problems of ineffective traffic flows, companies like Google, TomTom, HERE Technologies are hugely investing to solve the problems related to traffic flows and provide a better service for their customers.

To simulate different mobility models, we have benefited from the open-source traffic simulation framework SUMO. Simulation is recognized to be one of the most beneficial tools for analyzing traffic flows. For instance, it would be very difficult, expensive and dangerous to set a traffic jam test in the real world. So, simulation of traffic is very cost-effective and helpful for studying traffic flows [2]. Here, the motorcycle mobility is modeled and its impact on the distinct types of vehicles traveling on the road is studied. This might be useful for many companies working on traffic flow issues. To this end, six different mobility models for motorcycles are devised and the impact of these models is explored on traffic flows. Different driving scenarios result in different traffic flows, speed, travel time, fuel consumption and emission of $\mathrm{CO}_{2}$.

The remainder of this paper is organized as follows. Section two deals with concepts concerning modeling motorcycle mobility. In the next section, we discuss simulation platform parameters and requirements. The results are evaluated in section four, where the impact of six mobility models for motorcycles is analyzed from efficiency and environmental aspects. Finally, a conclusion and future work are provided in the last section.

\section{Mobility Modelling for Motorcycles}

This section addresses the basic concepts of mobility modeling for motorcycles. Some paradigms such as lane changing models, car following and emission models are described in detail and the most appropriate parameters will be selected for modeling. Later, six different mobility models (sublane, lane change, normal, acceleration and deceleration, incident, and density) are presented in the form of six scenarios.

Each scenario contains some specific parameters. Acceleration and deceleration values, length, width and other parameters of each vehicle type are simulated based on measurements and values that are available in the SUMO website [3]. Lane-changing models, car-following models and emission classes are explained as follows.

- Lane-Changing Model: three lane-changing models can be implemented in a SUMO simulation:

- LC2013: this lane-changing model is the default model and has been gener- 
ated by Jakob Erdmann based on the DK2008 model.

- SL2015: it is the lane-changing model that can simulate the mobility in a shared lane. In our research, this model is implemented especially in the sublane scenario to support the mobility of motorcycles in a shared lane.

- DK2008: this is the original lane-changing model of SUMO until version 0.18.0 which was generated by Daniel Krajzewicz.

- Car-Following Model: a car-following model simulates the mobility of a following vehicle with respect to the maneuver of the leading vehicle [2] [4] [5] [6]. Fifteen car following models are available on the SUMO website [3]. The three most popular car-following models are as follows:

- Krauss (the default car-following model of SUMO).

- Intelligent Driver Model (IDM).

- Wiedemann.

The Krauss model is a collision free model which means that no accident occurs when this model is used [2]. Krauss calculates the acceleration of a following vehicle and directly calculates the velocity instead of obtaining it from the acceleration value.

IDM calculates the speed directly based on the distance to the leading vehicle. This method is closer to the behavior of a driver in the real world [5].

The Wiedemann model is a psycho-physical distancing model. If a faster vehicle is approaching a slower leading vehicle, it will start to decelerate until it reaches its own threshold [2].

The information about speed and acceleration of the front vehicle are used in the Krauss and Wiedemann models which are usually difficult for a real driver to be calculated. This means that the Krauss and Wiedemann models react quicker than a human driver based on the acceleration and speed of the front vehicle. On the other hand, IDM calculates the speed based on the distance to the leading vehicle which is closer to the behavior of a driver in the real world [2] [5]. Since the IDM model is closer to the real world and a human driver behavior, it is the carfollowing model considered in our research.

- Emission Models: SUMO includes the following emission models [3] defining its own emission classes:

- HBEFA v2.1-based.

- HBEFA v3.1-based.

- PHEMlight.

- Electric Vehicle Model.

HBEFA v2.1-based: The Handbook Emission Factors for Road Transport (HBEFA) provides emission factors for all current vehicle types [3]. The vehicle classes supported by HBEFA have been classified. HBEFA was developed on behalf of the environmental protection agencies of Germany, Switzerland and Austria. Also, more countries (Sweden, Norway, and France) in addition to the JRC (European Research Center of the European Commission) are supporting HBEFA [7]. 
HBEFA v3.1-based: The vehicle classes in SUMO are mapped to the vehicle classes which are provided by HBEFA. This classification is the main difference between HBEFA 3.1 and HBEFA 2.1. It should be considered that these classes are collections of more specific vehicle classes in HBEFA [3].This type of emission model is used in our research.

PHEMlight. Passenger Car and Heavy-Duty Emission Model (PHEM) is a vehicle emission model that has been developed by the Technical University of Graz since 1999. PHEM is based on the measurements of a pervasive type of European vehicles which includes light vehicles like private car and heavy-duty vehicles such as bus and truck.

Electric Vehicle Model: SUMO has contained a model for electric vehicles since version 0.24 . The outputs of emission model of SUMO can be used for electric vehicles [3].

Considering the mentioned topics, six distinct scenarios are proposed as follows to model various driving patterns of motorcycles on the road:

- Sublane: One of the unique characteristics of motorcycles is their ability to share or overtake a vehicle in the same lane. The width of each lane is four meters (which is the standard width of one lane in Germany). To achieve the mentioned capability, each lane is divided into two 2-meter sublanes so that motorcycles can ride beside another vehicle in a shared lane.

- Lane Change: Motorcycles can easily change lane due to their particular dynamics and structure. Lane change command is sent to the motorcycles at a specific point in time to study the behavior of vehicular traffic accordingly.

- Normal: This acts a reference for other scenarios such that their results are to be evaluated with respect to the scenario. In the normal scenario, vehicles are inserted and travel on the road normally without any special maneuver or diversion.

- Acceleration and Deceleration: As one of the most important mobility features, motorcycles can accelerate or decelerate irregularly and suddenly. Therefore, to simulate this behavior, we let them accelerate and decelerate twice at two different times and positions on the road to discover to what extent they influence the mobility of other road users.

- Incident: In order to represent and analyze the impact of traffic jams caused by motorcycles on other vehicles, we made an occurrence happen to some of the motorcycles on the road and they stop in the middle of the route.

- Density: To show the impact of motorcycles density on vehicular traffic, motorcycles are forced to travel slower than other vehicles in the middle of the route in this scenario. This issue will affect the mobility of other vehicles and create congestion on the road.

\section{Implementation}

To simulate different scenarios of traffic flows and evaluate the impact of motorcycles on traffic flows, SUMO has been used. It has been developed by the German Aerospace Center (DLR) [3] and is available since 2001 allowing the simu- 
lation of traffic models. It includes roads, vehicles, public transport and pedestrians. SUMO supports tools for evaluating various outputs such as emissions. An overview of the graphical SUMO interface can be seen in Figure 1.

SUMO supports the programming languages Java, Python and C++. The Traffic Control Interface (TraCI) is one of the useful tools of SUMO which is used to send commands to the vehicles and manipulate their maneuvers when they are traveling on the route. TraCI uses a TCP-based connection to provide access to SUMO which acts as a server that is started with an additional command line.

In the implementation, $\mathrm{TraCI}$ has been used to control the mobility of vehicles especially in incident and lane change scenarios. SUMO only prepares the simulation and waits for external applications to connect and take over the control. After its start, clients connect to it by setting up a TCP connection to the appointed SUMO port. The client application sends commands to SUMO to control the simulation execution, influence single vehicle's behavior or to ask for environmental details. SUMO answers with a status-response to each command and additional results that depend on the given command. For example, in the incident and change lane scenario, a stop command and a changing lane command will be generated and sent through $\mathrm{TraCI}$ to the according vehicles.

In the simulation, four types of vehicles are considered. In total, 243 vehicles departed and traveled on the route. The detailed number of each vehicle type can be seen in Table 1. The length of the route is one kilometer including three edges (three parts) so that a more detailed investigation can be performed.

Here, our simulation contains some XML files for each scenario, one configuration file and python codes. By running the python codes, the SUMO software is launched. Then, by starting the simulation for 150 seconds, vehicles are inserted and travel on the road. During running the simulation, some output files are generated which are used to extract the average speed, travel time, $\mathrm{CO}_{2}$ emission and fuel consumption of different road users in various scenarios.

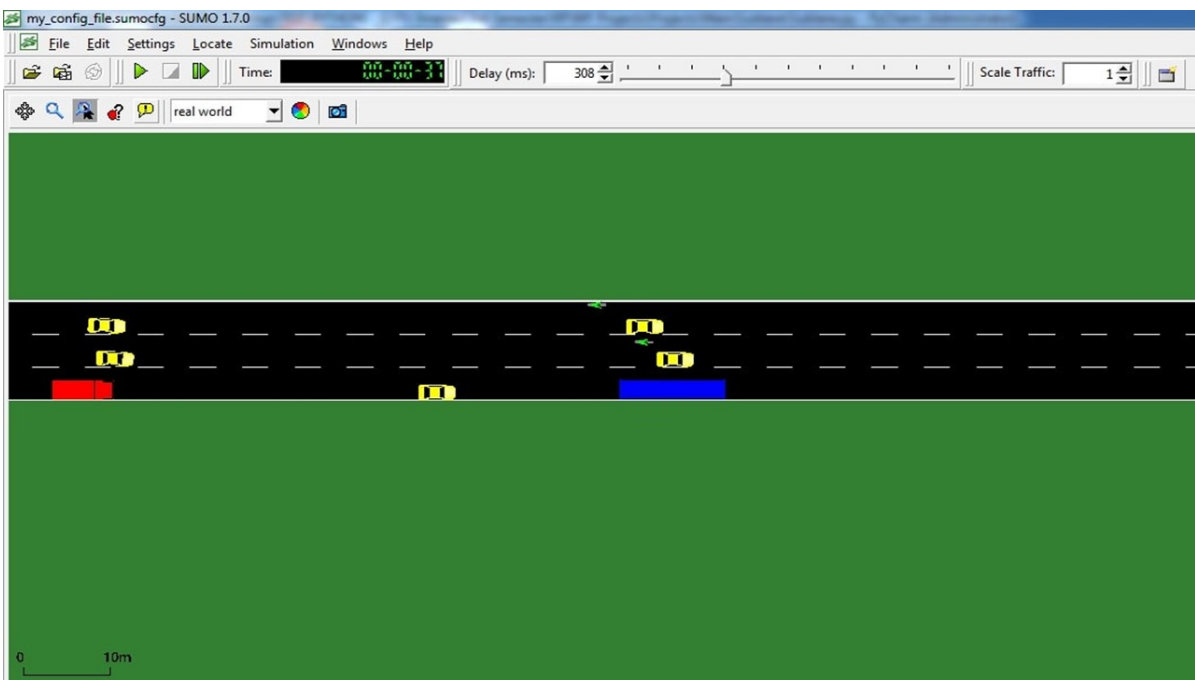

Figure 1. Graphical interface of SUMO. 
Table 1. Number of vehicles.

\begin{tabular}{cc}
\hline Vehicle Type & No. of Vehicles \\
\hline Bus & 26 \\
Car & 107 \\
Motorcycle & 84 \\
Truck & 26 \\
Total & 243 \\
\hline
\end{tabular}

\section{Evaluation}

In this section, six scenarios encompassing sublane, lane change, normal, acceleration/deceleration, incident and density for motorcycle mobility are taken into consideration. Next, the impact of these diverse scenarios on various types of vehicles in terms of average speed, travel time, fuel consumption and environmental sustainability is demonstrated. Moreover, it is shown how these different mobility patterns affect the vehicular traffic as a whole.

\subsection{Average Speed}

The average speed is calculated for each type of vehicle in all scenarios. Based on the simulation results in SUMO, average speed is obtained in each scenario for each type of vehicle during the time that vehicles are travelling on the route. This enables us to compare the average speed of different vehicles in every scenario. Moreover, the impact of motorcycles mobility can be deduced and compared through all scenarios. The average speed of different types of vehicles in each scenario is depicted in Figure 2.

As it can be seen in Figure 2, the average speed remained identical for sublane and lane change scenarios for different vehicles except for the motorcycles that showed a significant increase in the sublane scenario, around $38 \mathrm{~km} / \mathrm{h}$. This is due to their ability to move in a shared lane beside another vehicle which allows them to travel at the highest average speed. Furthermore, from normal to the density scenario, we witnessed a dramatic decline in the average speed of cars and motorcycles till the incident model where it leveled off to the density model. On the other hand, the average speed of heavy vehicles dropped with less steep over the last four scenarios. Although heavy vehicles traveled with lower average speeds for the first four mobility models, they experienced higher speeds than cars and motorcycles for the last two models. It can be concluded from the figure that density, incident and acceleration/deceleration scenarios had the highest impact on the average speed of vehicular traffic flow respectively. Besides, sublane and lane change models showed to have no negative impact on the vehicular mobility.

\subsection{Average Travel Time}

The average travel time is obtained based on the average travel time of each 


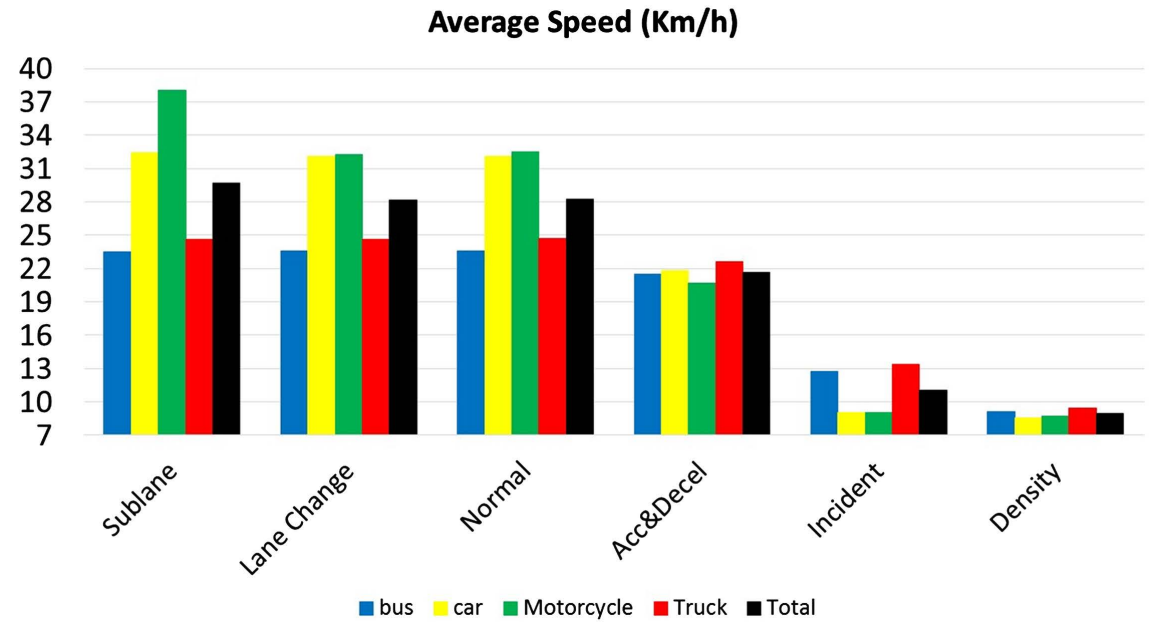

Figure 2. Average speed.

vehicle on the route in the simulation. In each scenario, travel time of each vehicle is calculated according to its departure and arrival time. Later, average travel time is performed for each type of vehicle. The line charts of the average travel time of all vehicles with respect to different mobility models of motorcycles can be seen in Figure 3. As it can be seen in the Figure, in regard to all vehicle types, the sublane scenario has the lowest average travel time while the density model has the highest value. Overall, compared to the normal scenario, except for the sublane and lane change scenarios, the average travel time increased rapidly over all other mobility models. Moreover, in case of sublane and lane change models, heavy vehicles reported slightly higher travel times while it was the other way around for the remaining scenarios. All in all, it is deduced that sublane and lane change mobility models can lower the average travel time of vehicular traffic whereas the other cases notably increase it.

\subsection{Average $\mathrm{CO}_{2}$ Emission}

As already discussed in the paper, each type of vehicle takes advantage of its own emission class. Sum of the $\mathrm{CO}_{2}$ emissions of each type of vehicle is computed during its travel time on the route. Afterwards, the average $\mathrm{CO}_{2}$ emission is achieved accordingly. Motorcycles mobility has different environmental impact on other vehicles in each scenario. In general, since truck and bus are in the Heavy Drive Vehicles (HDV) category in the emission class, they emit a higher amount of $\mathrm{CO}_{2}$ than other vehicle types in all scenarios.

Based on the illustrated emission of $\mathrm{CO}_{2}$ in Figure 4, the truck emission in the sublane, lane change and normal scenarios remained steady and reached the highest rate of emission, around $3.25 \mathrm{~kg}$ followed by the bus with $2.75 \mathrm{~kg}$. After that, the average $\mathrm{CO}_{2}$ emission for both heavy vehicles decreased marginally until it dipped suddenly in density mobility scenario to approximately 2.25 and $1.75 \mathrm{~kg}$ for truck and bus respectively.

In terms of car and motorcycle, they incurred less air pollution than heavy 


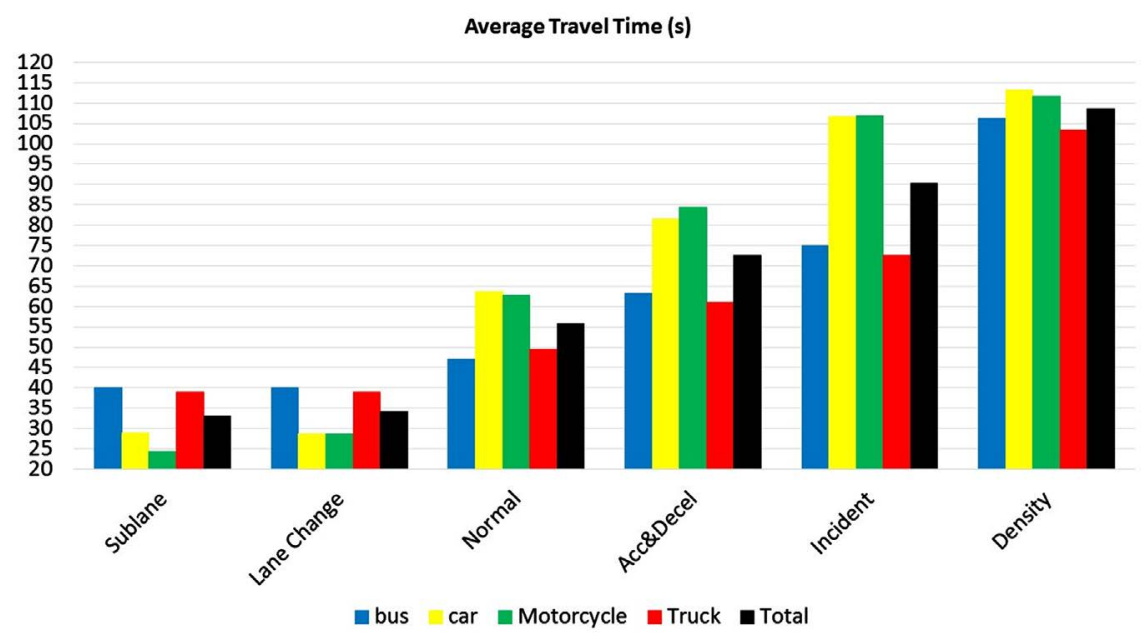

Figure 3. Average travel time.

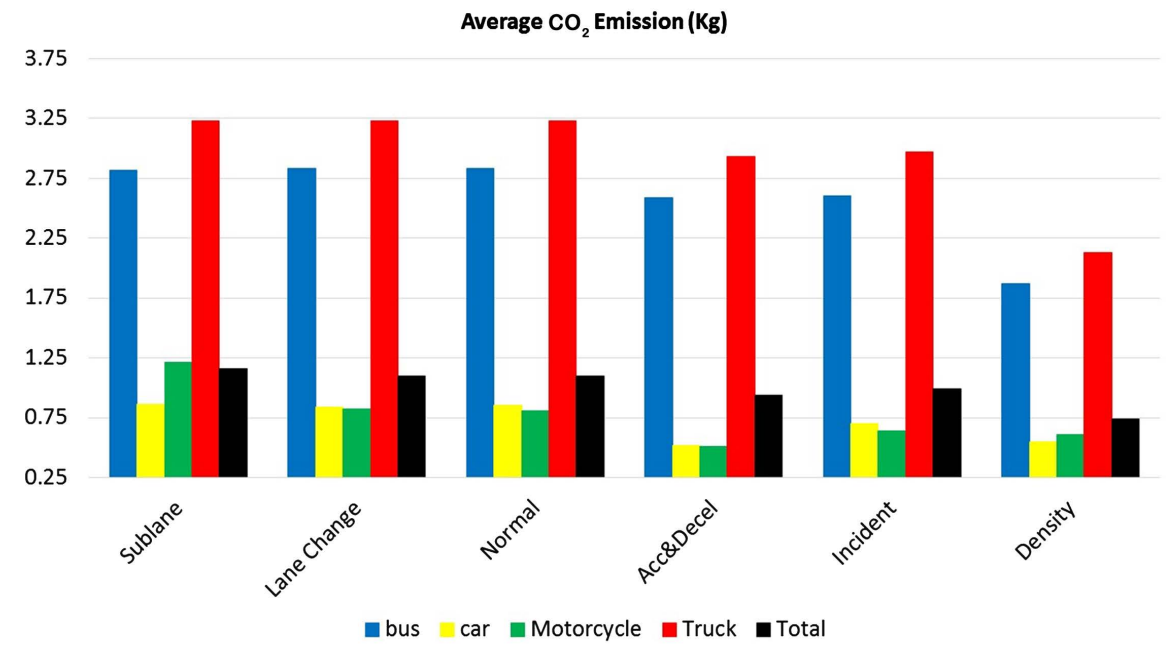

Figure 4. Average $\mathrm{CO}_{2}$ emission.

vehicles. The cars' average $\mathrm{CO}_{2}$ emission was nearly $0.75 \mathrm{~kg}$ for all mobility models except for the acceleration/deceleration case where the emission slightly fell to around $0.50 \mathrm{~kg}$. In addition, motorcycles showed the same behavior as cars in terms of $\mathrm{CO}_{2}$ emission apart from the sublane scenario where they emitted considerably more pollution than cars, roughly 1.5 times with almost $1.25 \mathrm{~kg}$ of $\mathrm{CO}_{2}$.

To sum up, it is derived that sublane and lane change scenarios had no particular impact on neither heavy nor low weight vehicles. The only exception is for motorcycles in the sublane model where their emission substantially climbed. Besides, other mobility models especially acceleration/deceleration for both cars and motorcycles and density for trucks and buses demonstrated to have reduced the ecological impact.

\subsection{Average Fuel Consumption}

The calculation of the average fuel consumption is akin to the calculation of the 


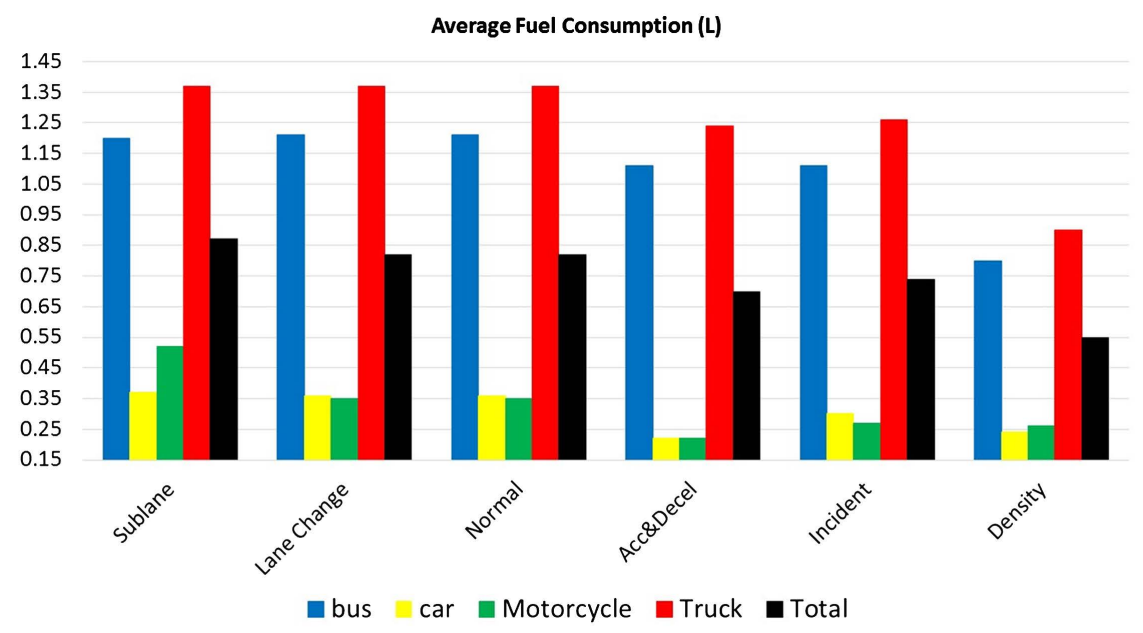

Figure 5. Average fuel consumption.

average $\mathrm{CO}_{2}$ emission that was previously discussed. The fuel consumption model is related to the emission class of each type of vehicle. A summation of the fuel consumption of each vehicle is done when the vehicle is moving on the route. Then, an average is computed to obtain the average fuel consumption for each type of vehicle. Figure 5 illustrates the average fuel consumption of all vehicle types in various mobility models. Basically, truck and bus had the highest fuel consumption among all vehicles, with the maximum of around 1.35 and 1.20 liters for the driven distance (one kilometer) respectively. Further, car and motorcycle consumed a similar and much lower amount of fuel with 0.55 and 0.35 liters at the peak and 0.24 liters at the lowest band.

As it can be seen in Figure 5, in comparison to the normal scenario, the average fuel consumption remained nearly unchanged through sublane and lane change models for all vehicles excluding motorcycles in the sublane scenario that consumed significantly more fuel. In all mobility models, the average fuel consumption diminished modestly in the acceleration/deceleration scenario where it grew faintly in the incident model before it fell drastically in the density scenario for heavy vehicles and a little for other vehicles.

\section{Conclusions and Future Work}

In this paper, the impact of motorcycles mobility on different classes of vehicles in six scenarios was investigated. Various mobility models of motorcycles and developed six major models including sublane, lane change, normal, acceleration/ deceleration, incident and density were studied. Furthermore, average speed, travel time, $\mathrm{CO}_{2}$ emission and fuel consumption were considered to evaluate the impact of motorcycles mobility on various kinds of vehicles such as motorcycles, cars, buses and trucks.

Based on the results, sublane and lane change scenarios showed to have neutral or positive impact on vehicular traffic in terms of the performance metrics of average speed, travel time, energy consumption and environmental sustai- 
nability. Moreover, acceleration/deceleration, incident and density mobility models proved to degrade the vehicular traffic flow in terms of average speed and travel time. In contrast and from ecological aspect, the aforementioned scenarios improved $\mathrm{CO}_{2}$ emission especially in acceleration/deceleration for low-weighted vehicles and in density model for heavy weighted ones. Therefore, it is concluded that depending on the mobility model of the motorcycle, vehicular traffic flow can be enhanced or deteriorated. In future work, we aim to incorporate pedestrian mobility to examine the impact of another vulnerable road user on vehicular traffic.

\section{Conflicts of Interest}

The authors declare no conflicts of interest regarding the publication of this paper.

\section{References}

[1] Codeca, L. and Härri, J. (2019) Powered Two-Wheelers Extensive Simulation Study in SUMO. EURECOM, Department of Communication Systems, Technical Report No. RR-19-340.

[2] Bieker-Walz, L., Behrisch, M., Junghans, M. and Gimm, K. (2017) Evaluation of Car-Following-Models at Controlled Intersections. 31 st European Simulation and Modelling Conference (ESM2017), Lisbon, Portugal, 25-27 October 2017, 247-251.

[3] (n.d.) Simulation of Urban Mobility. https://www.eclipse.org/sumo.

[4] Zhu, M.X., Wang, X.S., Tarko, A. and Fang, S. (2018) Modeling Car-Following Behavior on Urban Expressways in Shanghai: A Naturalistic Driving Study. Transportation Research Part C: Emerging Technologies, 93, 425-445. https://doi.org/10.1016/j.trc.2018.06.009

[5] Pourabdollah, M., Bjärkvik, E., Fürer, F., Lindenberg, B. and Burgdorf, K. (2017) Calibration and Evaluation of Car Following Models Using Real-World Driving Data. 2017 IEEE 20 th International Conference on Intelligent Transportation Systems (ITSC), Yokohama, Japan, 16-19 October 2017, 1-6. https://doi.org/10.1109/ITSC.2017.8317836

[6] Erdmann, J., Niebel, W., Krajzewicz, D., Härri, J. and Bensator, S. (n.d.) Cooperative Self-Organizing System for Low Carbon Mobility at Low Penetration Rates. https://cordis.europa.eu/project/id/318622

[7] (n.d.) Handbook Emission Factors for Road Transport (HBEFA). https://www.hbefa.net/e/index.html 